EPJ Web of Conferences 88, 00002 (2015)

DOI: $10.1051 /$ epjconf/ 20158800002

(C) Owned by the authors, published by EDP Sciences - SIF, 2015

\title{
Nuclear thermodynamics and isospin degree of freedom
}

\author{
M.-F. RIVET \\ Institut de Physique Nucléaire, CNRS/IN2P3, Université Paris-Sud 11, \\ 91406 Orsay, France
}

\begin{abstract}
Nuclear thermodynamics studies evidenced the existence of a liquid-gas type phase transition, that manifests through multifragmentation and may originate from spinodal instabilities. Several signals typical of finite systems were experimentally observed. The spinodal region of the phase diagram is theoretically predicted to shrink for systems away from the valley of stability. What happens to the signals of phase transition for such systems should thus bring further information on the symmetry energy term of the nuclear equation of state.
\end{abstract}

\section{Introduction}

During the last decades nuclear thermodynamics was widely studied through heavy-ion collisions during which a nucleus (or a nuclear system) can be heated, compressed, diluted These systems are expected to undergo a liquidgas type phase transition, due to the analogy between the nuclear interaction and the force acting on Van der Waals fluids, that manifests through nuclear multifragmentation. Finite systems such as nuclei show specific behaviours in the transition region. Most of the predicted specific signals were experimentally evidenced, they indicate that multifragmentation may be induced by spinodal instabilities $[1,2]$. This happens when the system evolves through the mechanically unstable spinodal region of the phase diagram, located at densities $\rho \leq \rho_{0}$ and temperature below the critical temperature. 
Such conditions are explored in central and mid-peripheral nuclear collisions around the Fermi energy. Theoretical works show that chemical and mechanical instabilities are closely related in the spinodal zone, the extension of which depends on the isospin of the nuclear system [3]. Therefore, thanks to the advent of exotic beams, present experimental and theoretical works focus on the role of isospin on nuclear thermodynamics, probing that way the symmetry energy term of the nuclear Equation Of State (EOS). Besides basic knowledge on the nuclear EOS, such experiments are important for studying the structure of exotic nuclei, neutron star crust, supernova explosions ...

In this paper, after a brief review of the nuclear phase transition signals we shall discuss some experimental results showing the influence of isospin on the caloric curve, on isospin distillation and on the level density parameter.

\section{Phase transition in finite systems}

Because in finite systems surface effects are not negligible with respect to bulk properties, the entropy of finite nuclear systems presents an abnormal convexity in the phase transition region; this behaviour induces specific transition signals:

- a bimodal distribution of extensive variables, for example the energy, or the charge of the largest fragment $\left(\mathrm{Z}_{\max }\right)$ of the multifragmentation partitions. Bimodality was observed in $60-100 \mathrm{AMeV} \mathrm{Au+Au} \mathrm{semi-}$ peripheral collisions, allowing to estimate the latent heat for nuclei close to gold around $8 \mathrm{MeV}$ [4].

- the dynamics of the phase transition proceeds through spinodal instabilities; the extra production of equal-sized fragment partitions supports this hypothesis $[5,6]$. In that case multifragmentation should be an aggregation phenomenon; this was proved by the observation of a universal $(\Delta)$-scaling law of the fluctuations of $Z_{\max }$, which is thus related to an order parameter $[7,8]$.

- entropy convexity is necessarily accompanied by a backbending of the caloric curve $T=f\left(E^{*}\right)$ and a negative heat capacity in the spinodal zone. The latter signal was early observed in $35 \mathrm{AMeV} \mathrm{Au+Au} \mathrm{semi-}$ peripheral collisions [9].

- experimentally determined caloric curves all present a similar shape whatever the mass of the system $(A=30-240)$, namely a Fermi gas 


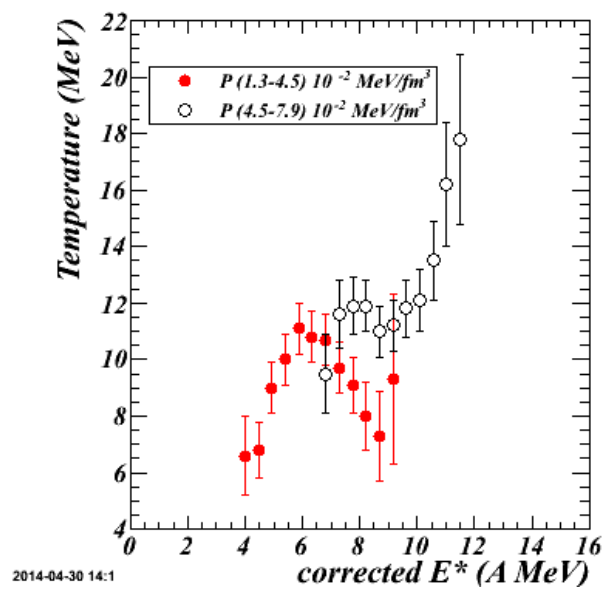

Figure 1: Caloric curves determined for heavy systems produced in central $\mathrm{Xe}+\mathrm{Sn}$ collisions for selected pressure intervals. Adapted from [14].

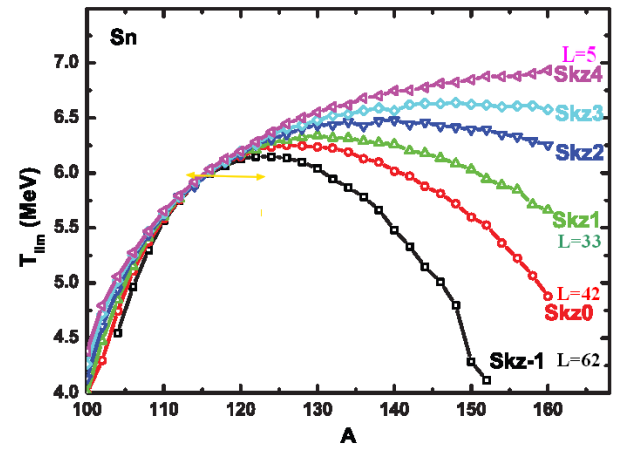

Figure 2: Limiting temperature vs the mass of Sn nuclei, calculated from EOS with different SkzN forces, characterized by the slope L of the symmetry term. Adapted from [15].

behaviour at low energy $(\mathrm{T} \propto \sqrt{E / A})$, followed by a plateau in the transition region then by a classical gas law $(\mathrm{T} \propto(E / A))$ at high energy. The temperature at the plateau, $T_{l i m}$, decreases and is reached at lower energy when the system mass increases $[10,11]$. The shape of the caloric curve depends however on the path followed by the system in the potential landscape, and a backbending can only be observed for a transition at constant pressure [12]. This was evidenced recently for central 32-50 $A \mathrm{MeV} \mathrm{Xe}+\mathrm{Sn}$ collisions, thanks to a simulation based on experimental data in which a quantal temperature was calculated from the momentum fluctuations of protons present at freeze-out [13]. Pressure-constrained caloric curves could be built that exhibit the expected backbending [14], as shown in fig. 1 .

\section{Equation of state of asymmetric nuclear matter}

The neutron to proton ratio of the studied nuclear systems may influence nuclear dynamics and thermodynamics through the symmetry energy term of the nuclear EOS :

$$
\varepsilon(\rho, I)=\varepsilon(\rho, I=0)+\varepsilon_{\text {sym }}(\rho) \times I^{2}+\mathcal{O}(4)
$$


with $I=\left(\rho_{n}-\rho_{p}\right) / \rho=(N-Z) / A$ and $\varepsilon=E / A$. A second order expansion of the symmetry term around normal density $\rho_{0}$ reads:

$$
\varepsilon_{\text {sym }}(\rho)=a_{4}+\frac{L}{3}\left(\frac{\rho-\rho_{0}}{\rho_{0}}\right)+\frac{K_{\text {sym }}}{18}\left(\frac{\rho-\rho_{0}}{\rho_{0}}\right)^{2}
$$

The slope $L$ characterizes the (asy)-stifness of the EOS. Isospin effects are expected to be small, as $I$ is always smaller than $1 ; I$ in the range $0-0.2$ for stable nuclei. In theories, spinodal instabilities arise from mechanical (density) and chemical (isospin) instabilities, both strongly tangled [16]. The spinodal zone is predicted to shrink for increasing isospin asymmetry, reducing both the critical density and temperature $[3,17]$. As spinodal instabilities are most probably at the origin of the phase transition (multifragmentation) any change in the spinodal domain of the phase diagram should affect the signals described in the previous section.

\section{Effects of isospin on ...}

In this section I will briefly review some isospin effects on phase transition signals that were experimentally observed in the past years.

\subsection{The caloric curve}

A few experiments studied the effect of isospin on caloric curves, considering semi-peripheral collisions. Sfienti et al. for $600 \mathrm{AMeV}$ $\left({ }^{124} \mathrm{Sn},{ }^{124} \mathrm{La},{ }^{107} \mathrm{Sn}\right)+\mathrm{Sn}[18]$ and Wuenschel et al. for $35 \mathrm{AMeV}{ }^{78} \mathrm{Kr}+{ }^{58} \mathrm{Ni}$, ${ }^{86} \mathrm{Kr}+{ }^{64} \mathrm{Ni}[19]$ found tiny dependence on the system isospin, with slightly higher temperatures for the neutron-richer systems; conversely McIntosh et al., for light quasi-projectiles of known $\mathrm{A}$ and $\mathrm{Z}$ formed in $35 \mathrm{~A} \mathrm{MeV}^{70} \mathrm{Zn}+{ }^{70} \mathrm{Zn},{ }^{64} \mathrm{Zn}+{ }^{64} \mathrm{Zn},{ }^{64} \mathrm{Ni}+{ }^{64} \mathrm{Ni}$ observed measurable effects, with lower temperatures for neutron-richer nuclei [20]. Note that, unlike the ensemble of caloric curves presented in [11], none of those derived in [18-20] exhibits a plateau. In [20] the temperature linearly increases with energy between 2 and $8 \mathrm{AMeV}$, reaching $12 \mathrm{MeV}$ at $E^{*}=8 \mathrm{AMeV}$, well above the empirical value of $T_{\text {lim }}$ for light nuclei estimated in [11].

On the theoretical side, the SMM model, as well as calculations considering a nucleus in equilibrium with its vapor, predict an increase of $T_{l i m}$ with isospin [21,22], whereas in calculations dealing with isolated mononuclei, without surrounding vapor, the reverse trend is obtained [23]. In all cases the temperature variation with isospin is small. Recently Li Ou et al. studied isobar distributions of $T_{\text {lim }}$ for tin nuclei. They use Skyrme forces with 


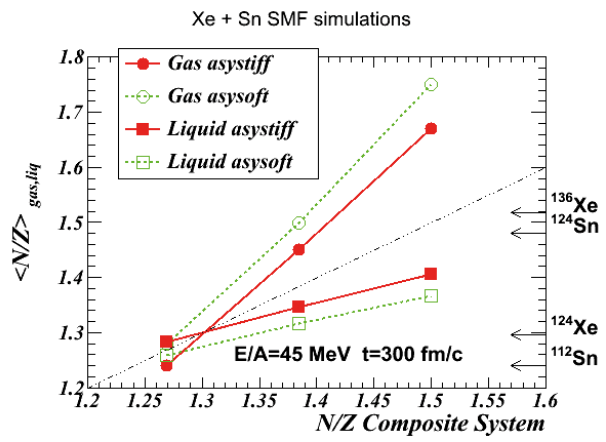

Figure 3: Evolution of $\mathrm{N} / \mathrm{Z}$ of primary fragments $v s$ that of the composite system for $\mathrm{Xe}+\mathrm{Sn}$ reactions at $45 \mathrm{AMeV}$, calculated with the SMF model using two asy-stiffnesses for the EOS.

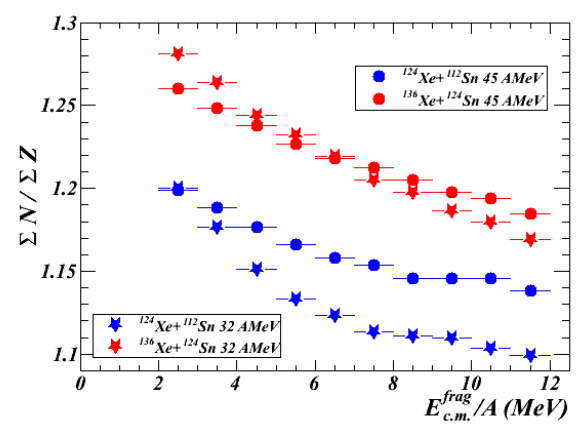

Figure 4: Evolution of the fragment $(Z>$ 3) N/Z vs their centre of mass kinetic energy per nucleon for $\mathrm{Xe}+\mathrm{Sn}$ reactions at 32 and $45 \mathrm{AMeV}$. Data from the INDRA Collaboration.

different asy-stiffnesses [24], in the framework of a nucleus in equilibrium with its vapor [15]. Results are shown in fig. 2. It clearly appears that the influence of the asy-stiffness of the EOS is small as long as Sn nuclei are "proton-rich" ( $A \leq 120$, i.e. the average stable Sn mass) and becomes sizeable on the neutron-rich side. Due to the combined effects of Coulomb and symmetry energy, $T_{\text {lim }}$ first increases with $A$ for low masses, before decreasing for very neutron-rich nuclei. This figure demonstrates how important it is to precisely know the isospin of the studied nuclei when establishing caloric curves.

\subsection{Isospin distillation}

In the phase transition domain, the liquid and gas phases coexist but have different $\mathrm{n} / \mathrm{p}$ ratios, because the gradients of the neutron and proton chemical potentials $v s$ density are opposite: this effect is called isospin distillation and signs the occurrence of the phase transition. An example is shown in fig. 3, obtained for Rf composite systems using the Stochastic Mean Field - SMF - simulations described in [25]. The difference between gas and liquid concentrations is larger when the EOS is asy-soft $(\mathrm{L}=49)$ than when the symmetry energy potential linearly varies with density (asy-stiff, $\mathrm{L}=75$ ). For heavy systems, the gas is more neutron-rich than the liquid when their $\mathrm{n} / \mathrm{p}$ ratio is large; conversely for less neutron-rich systems $(\mathrm{N} / \mathrm{Z}=1.25-1.3)$ the two phases present almost the same $\mathrm{N} / \mathrm{Z}$. As for caloric curves, we observe that more information on isospin effects can be expected from the study of 
very neutron-rich systems.

Studying the $\mathrm{N} / \mathrm{Z}$ of the fragments produced through multifragmentation in central collisions vs their kinetic energy should allow to quantify the strength of the symmetry term: the slope of $(N / Z)_{\text {frag }}$ vs $E_{k i n}$ is governed by the competition between Coulomb force - that accelerates more the proton-rich fragments, giving a negative slope - and the symmetry energy more repulsive for neutron-rich fragments, positive slope. In SMF simulations a negative slope is observed for $50 A \mathrm{MeV}{ }^{112,124} \mathrm{Sn}+{ }^{112,124} \mathrm{Sn}$ while a null or slightly positive one shows up for ${ }^{132} \mathrm{Sn}+{ }^{132} \mathrm{Sn}$. The effect, although attenuated, survives secondary decay [26]. Again a significant effect is visible when going to very neutron-rich systems. This variable was tested by the MSU group on $50 \mathrm{AMeV}{ }^{112,124} \mathrm{Sn}+{ }^{112,124} \mathrm{Sn}$ reactions [27] and by the INDRA collaboration for $32,45 A \mathrm{MeV}{ }^{124,136} \mathrm{Xe}+{ }^{112,124} \mathrm{Sn}$ systems. Both series of data are in agreement at 45, $50 \mathrm{AMeV}$, in shape and magnitude. Fig. 4 shows the INDRA data, which, as predicted, display a less negative slope for the neutron-rich system. For the "proton-rich system" ${ }^{124} \mathrm{Xe}+{ }^{112} \mathrm{Sn}$ the values of $(N / Z)_{\text {frag }}$ are much smaller at $32 \mathrm{AMeV}$ than at $45 \mathrm{AMeV}$ , with a more negative slope, confirming the importance of the Coulomb force with little flow. Conversely the results for the neutron-rich system ${ }^{136} \mathrm{Xe}+{ }^{124} \mathrm{Sn}$ are rather similar in magnitude at both energies, again with a slightly more negative slope at $32 \mathrm{AMeV}$. A quantitative comparison with SMF results is not relevant, as this simulation underestimates the fragment kinetic energy [28]. New calculations should be performed with the more advanced simulation BLOB [29].

\subsection{Level density parameter}

The last point I want to discuss is the evolution of the level density parameter with isospin. This parameter, $a$, is a direct property of the EOS, and was shown to depend on the temperature of the nucleus. An additional, and small, isospin dependence is also expected from the Fermi gas model and Thomas-Fermi calculations. A larger dependence may arise from the effective nucleon masses, and also for nuclei at large excitation energy or close to a drip line. Yet when isospin-dependent dynamical simulations are coupled to the de-excitation of hot primary products with a statistical code, the dynamical EOS and the level density in the after-burner are disconnected.

Empirical evolutions of $a$ with isospin on a large nucleus range were derived by Al-Quraishi et al. in 2001 and 2003 [30,32]. They fit the body 


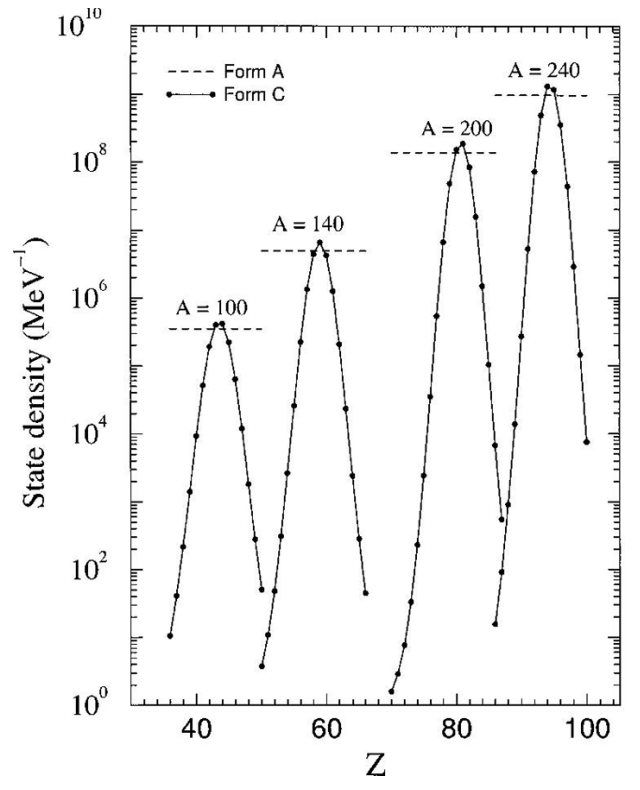

Figure 5: Evolution of the level density $v s$ the atomic number for different masses, following equations $\mathrm{A}$ and $\mathrm{C}$. From [30].

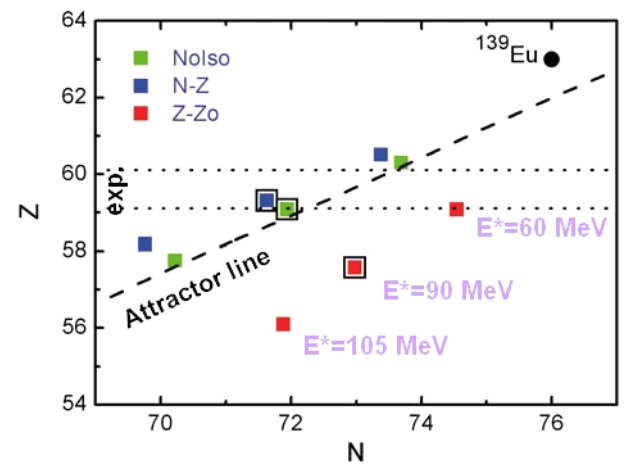

Figure 6: Average charge of evaporation residues as a function of their neutron number, calculated within LILITA_N97(see text) for de-excitation of ${ }^{139} \mathrm{Eu}$ at $E^{*}=60,90$ and $105 \mathrm{MeV}$. Dashed lines indicate the experimental data. Adapted from [31].

of measured level densities with different formulae:

$$
\begin{aligned}
a & =\alpha A \\
a & =\alpha A / \exp \beta(N-Z)^{2} \\
a & =\alpha A / \exp \gamma\left(Z-Z_{0}\right)^{2}
\end{aligned}
$$

Form (A) has no isospin dependence, (B) presents a moderate dependence on the neutron excess while form $(\mathrm{C})$ rapidly decreases for isotopes out of the valley of stability and even cancels for very exotic nuclei, as appears in fig. 5. The best value of $\alpha$ was 0.1 in the three cases.

In a recent experiment Moro et al. studied the de-excitation of the proton-rich nucleus ${ }^{139} \mathrm{Eu}$ formed at an excitation energy of $90 \mathrm{MeV}$; they measured the cross-sections of evaporation residues and the multiplicities of evaporated protons and $\alpha$-particles. They compared the data with the results of the statistical code LILITA-N97, implemented with the three forms of level densities proposed above [31]. Experimental and calculated results for residues are shown in fig. 6. The conclusion was that form (C) is completely ruled-out while forms (A) and (B) lead to close results compatible with experiment; proton and $\alpha$ multiplicities add a further constraint that 
slightly favours form (B). The same conclusion was derived by Charity et al. when studying $\mathrm{Yb}$ nuclei excited between 90 and $250 \mathrm{MeV}$ [33]. Results concerning ${ }^{92-104} \mathrm{Pd}$ nuclei excited at $2.9 \mathrm{~A} \mathrm{MeV}$ obtained from a fully exclusive experiment should bring additional information [34, and G. Ademard this conference].

\section{Conclusions}

Isospin effects on nuclear thermodynamics at subnormal densities and incident energies lower than $100 \mathrm{AMeV}$ are theoretically predicted; they should be more visible for neutron-rich nuclei as Coulomb effects impede those of the symmetry potential. The hot nucleus asymmetry is however uncertain in experiments, due to preequilibrium emission and evaporation, which lead for instance to contradictory observations concerning isospin effects on caloric curves. It appears important to master the variation of the level density of nuclei with asymmetry. Indeed in theoretical calculations there is no consistency between the nuclear EOS (in transport codes) and the EOS-dependent level density parameter (de-excitation codes). Experimental results are scarce, new information would be welcome.

In this context, to really gain information it is mandatory to have available facilities accelerating exotic neutron-rich nuclei (namely fission fragments of uranium) up to at least $50 \mathrm{AMeV}$.

\section{References}

[1] P. Chomaz, M. Colonna and J. Randrup, Phys. Rep. 389 (2004) 263.

[2] B. Borderie and M. F. Rivet, Prog. Part. Nucl. Phys. 61 (2008) 551.

[3] M. Colonna, P. Chomaz and S. Ayik, Phys. Rev. Lett. 88 (2002) 122701.

[4] E. Bonnet, D. Mercier et al. (INDRA Collaboration), Phys. Rev. Lett. 103 (2009) 072701.

[5] B. Borderie, G. Tăbăcaru et al. (INDRA Collaboration), Phys. Rev. Lett. 86 (2001) 3252.

[6] G. Tăbăcaru, B. Borderie et al., Eur. Phys. J. A 18 (2003) 103.

[7] J. D. Frankland, A. Chbihi et al. (INDRA and ALADIN collaborations), Phys. Rev. C 71 (2005) 034607. 
[8] D. Gruyer, J. Frankland et al. (INDRA Collaboration), Phys. Rev. Lett. 110 (2013) 172701.

[9] M. D'Agostino, R. Bougault et al., Nucl. Phys. A 699 (2002) 795.

[10] J. Pochodzalla, T. Mhlenkamp et al. (ALADIN Collaboration), Phys. Rev. Lett. 75 (1995) 1040.

[11] J. B. Natowitz, K. Hagel et al., Phys. Rev. Lett 89 (2002) 212701.

[12] P. Chomaz, V. Duflot and F. Gulminelli, Phys. Rev. Lett. 85 (2000) 3587.

[13] H. Zheng and A. Bonasera, Phys. Lett. B 696 (2011) 178.

[14] B. Borderie, S. Piantelli et al. (INDRA Collaboration), Phys. Lett. B 723 (2013) 140.

[15] Li Ou, Min Liu and Zhuxia Li, Phys. Rev C 89 (2014) 011001.

[16] H. Mller and B. D. Serot, Phys. Rev. C 52 (1995) 2072.

[17] V. Baran, M. Colonna et al., Nucl. Phys. A 632 (1998) 287.

[18] C. Sfienti, P. Adrich et al. (ALADIN2000 Collaboration), Phys. Rev. Lett. 102 (2009) 152701.

[19] S. Wuenschel, A. Bonasera et al., Nucl. Phys. A 843 (2010) 1.

[20] A. B. McIntosh, A. Bonasera et al., Phys. Lett. B 719 (2013) 337.

[21] R. Ogul, A. S. Botvina et al., Phys. Rev. C 83 (2011) 024608.

[22] J. Besprovany and S. Levit, Phys. Lett. B 217 (1989) 1.

[23] C. Hoel, L. G. Sobotka and R. J. Charity, Phys. Rev. C 75 (2007) 017601.

[24] J. Margueron, J. Navarro and Nguyen Van Giai, Phys. Rev. C 66 (2002) 014303.

[25] M. Colonna, M. Di Toro et al., Nucl. Phys. A 642 (1998) 449.

[26] M. Colonna, V. Baran et al., Phys. Rev. C 78 (2008) 064618.

[27] T. X. Liu, W. G. Lynch et al., Phys. Rev. C 86 (2012) 024605. 
[28] F. Gagnon-Moisan, E. Galichet et al. (INDRA Collaboration), Phys. Rev. C 86 (2012) 044617.

[29] P. Napolitani and M. Colonna, Phys. Lett. B 726 (2013) 382.

[30] S. I. Al-Quraishi, S. M. Grimes et al., Phys. Rev. C 63 (2001) 065803.

[31] R. Moro, A. Brondi et al., Eur. Phys. J. A 48 (2012) 158.

[32] S. I. Al-Quraishi, S. M. Grimes et al., Phys. Rev. C 67 (2003) 015803.

[33] R. J. Charity, L. G. Sobotka et al., Phys. Rev. C 67 (2003) 044611.

[34] P. Marini, M. F. Rivet et al. (INDRA collaboration), Proc. II Int. Workshop on Compound Nucleus Reactions, EDP Sciences, 2010, vol. 2 of EPJ Web of Conferences, 04003, http://dx.doi.org/10.1051/epjconf/20100204003. 